\title{
Comparação de gráficos de controle univariados e multivariados aplicados a dados de instrumentação da usina hidrelétrica de Itaipu
}

\author{
Emerson Lazzarotto, \\ Centro de Engenharias e Ciências Exatas, Universidade Estadual do Oeste do Paraná \\ Dinter PPGMNE/UFPR-Unioeste-PTI, 85.870-650, Foz do Iguaçu - PR \\ e-mail: emerson.lazzarotto@gmail.com, emerson.lazzarotto@unioeste.br
}

\section{Liliana Madalena Gramani, Anselmo Chaves Neto}

Universidade Federal do Paraná - Setor de Ciências Exatas, Departamentos de Matemática e Estatística PPGMNE - Centro Politécnico, Curitiba - PR

e-mail:1.gramani@gmail.com, anselmo@ufpr.br

\begin{abstract}
Resumo: Os instrumentos de monitoramento de barragens em usina hidrelétricas fornecem extensas bases de dados das quais podem ser retiradas importantes informações. Uma técnica adequada para tal são os gráficos de controle estabelecidos com base em critérios estatísticos. Neste trabalho é realizada a interpretação e a comparação entre gráficos de controle básicos, os gráficos univariados $\bar{x}$ e os gráficos bivariados $T^{2}$ de Hotelling aplicados aos dados de leitura de piezômetros localizados no trecho $E$ da barragem da usina hidrelétrica de Itaipu. Resultados demonstram que os gráficos multivariados são capazes de reduzir a taxa de falsos alarmes, facilitar a identificação de outliers, apontar uma das principais causas de variabilidade nestes gráficos de controle e estabelecer limites de controle operacionais para as leituras de instrumentos da usina.
\end{abstract}

\section{Introdução}

Devido à importância econômica e social das usinas hidrelétricas é fundamental que estas possuam, dependendo do seu tamanho, uma extensa variedade e quantidade de instrumentos de monitoramento para acompanhar a segurança do seu processo de operação e servir de alerta para situações de anormalidade.

Há diversos instrumentos para avaliar o comportamento da barragem de uma usina, dentre eles: pêndulos, termômetros, extensômetros, piezômetros, tensômetros, entre outros. Normalmente, usinas de médio e grande porte, já possuem monitoramento on-line de seus instrumentos, recebendo com alta freqüência uma grande massa de dados proveniente destes instrumentos.

As séries históricas de dados de leitura de instrumentos funcionando na fase de operação da usina são objeto de estudos estatísticos como séries temporais, gráficos (cartas) de controle que tem como principal objetivo acompanhar o comportamento da barragem e fornecer uma ferramenta adicional à equipe de auscultação na indicação de alertas e na avaliação da estabilidade do processo.

Neste contexto, algumas questões são colocadas: existe correlação entre instrumentos com a mesma finalidade ou finalidade distinta? Quais fatores (variáveis) ambientais afetam a variabilidade de um dado instrumento? Qual o grau desta influência? É possível reduzir a taxa de alertas estatísticos falsos?

A análise individual desta grande quantidade de dados de instrumentos, além de ser laboriosa, pode gerar falsos alarmes (por exemplo, instrumentos defeituosos, mudança no valor médio). Johnson[6], Montgomery[7] e Ryan[9] consideram que um avanço no controle estatístico da instrumentação de barragens pode se dar por meio do uso de técnicas multivariadas. A análise multivariada pode explicar as principais causas atribuíveis de variabilidade na leitura de dados de instrumentos de monitoramento de barragem, reduzir a taxa de alertas falsos, ou seja, de forma geral, promover uma melhoria na qualidade do controle.

Mais recentemente, Cheng [4] propôs dois modelos que buscam explicar a relação entre as variáveis ambientais e dados de instrumentos de monitoramento de barragens através da análise 
da matriz de covariância de um determinado instrumento, determinação de componentes principais e gráficos de controle para avaliar se novos dados apresentam comportamento normal, Yu et al.[11] combinam um modelo de extração de componentes principais de conjunto de dados com um modelo hidro-sazonal no tempo para estabelecer gráficos de controle e fazer previsões de dados futuros, Gu et al. [5] também fazem um estudo de caso em que, a partir da análise das componentes principais, estabelecem gráficos de controle $T^{2}$ de Hotelling para monitorar a segurança de uma barragem obtendo melhoria na redução de dados, filtro de ruído e diminuição da taxa de falsos alarmes.

Neste trabalho é feita uma comparação entre a taxa de falsos alarmes gerados através da utilização de cartas de controle univariadas e sua generalização em termos de cartas de controle bivariadas aplicadas ao caso de um instrumento da usina hidrelétrica de Itaipu.

\section{Materiais e métodos}

Myers [8] aponta que as três componentes críticas de um sistema de monitoramento de barragem são: instrumentação, coleta e o manejo dos dados e que é objetivo a contínua otimização do sistema de monitoramento. Para tal, o recebimento de dados através de equipamentos automatizados de coleta de dados tem contribuído para o aumento da confiabilidade dos dados, a apresentação de resultados em tempo real e a busca de novos conhecimentos que podem ser extraídos a fim de identificar mudanças e facilitar ações corretivas.

No contexto destes aspectos de apresentação de resultados em tempo real e a busca por novos conhecimentos, os gráficos de controle tem se mostrado uma ferramenta simples e eficaz de realizar o controle estatístico de um processo, no caso, o processo de monitoramento da instrumentação de barragens.

Montgomery [7] relata três usos fundamentais dos gráficos de controle: redução da variabilidade do processo, monitoramento e vigilância do processo e estimação de parâmetros do processo. Cita ainda que algumas das razões de sua popularidade (aplicáveis ao caso de monitoramento de barragens) são a sua eficiência na prevenção de defeitos, que evitam ajustes desnecessários do processo e que fornecem informações de diagnóstico. Os gráficos de controle estatísticos univariados foram propostos por W.A. Shewhart e seu modelo geral consiste em determinar um intervalo ao redor da média de uma característica de qualidade de interesse.

Considere que $p$ variáveis representem processos que estejam sendo controladas, no caso, leituras de monitoramento de instrumentos, assumindo que $\underline{\boldsymbol{X}}^{\prime}=\left[X_{1}, X_{2}, \ldots, X_{p}\right] \sim N_{p}(\underline{\mu}, \Sigma)$, ou seja, que $\underline{X}^{\prime}$ tenha distribuição normal multivariada e que se deseja controlar a média $\mu$ do processo, bem como as covariâncias entre as variáveis $X_{i}$ e as variâncias $V\left(X_{i}\right)$. A alteração de ao menos uma das médias ou das covariâncias (variâncias) significaria que o processo está fora de controle. Neste caso, Montgomery [7] e Johnson [6] relatam que a aplicação de gráficos de controle univariados pode conduzir a interpretações equivocadas e enganosas e que os métodos multivariados são uma boa alternativa. Ryan [9] afirma ainda que quando as variáveis são correlacionadas aumenta a probabilidade de emissão de falsos alarmes ou, pior ainda, de não receber um sinal de alerta quando o processo multivariado esteja fora de controle.

A estatística $T^{2}$ introduzida por Hotteling pode ser considerada uma generalização da estatística $t$ onde

$$
t=\frac{\bar{x}-\mu}{s / \sqrt{n}}
$$

possui distribuição $t$, quando se deseja testar a hipótese $\mu=\mu_{0}$ tem-se que

$$
t^{2}=\frac{\left(\bar{x}-\mu_{0}\right)^{2}}{s^{2} / n}=n\left(\bar{x}-\mu_{0}\right)\left[s^{2}\right]^{-1}\left(\bar{x}-\mu_{0}\right) \text {. }
$$

Logo, no caso $p$ dimensional segue que

$$
T^{2}=n\left(\overline{\boldsymbol{x}}-\boldsymbol{\mu}_{0}\right)^{\prime}[S]^{-1}\left(\overline{\boldsymbol{x}}-\boldsymbol{\mu}_{0}\right) .
$$

$S^{-1}$ é a inversa da matriz de covariância (os elementos da diagonal principal são as variâncias e os elementos fora da diagonal principal são as covariâncias entre as $p$ variáveis). 
No caso de leituras de instrumentação, as amostras extraídas para avaliação são de tamanho $n=1$, se $p$ instrumentos estiverem sendo avaliados, então a estatística torna-se

$$
T^{2}=\left(\overline{\boldsymbol{x}}-\boldsymbol{\mu}_{0}\right)^{\prime}[S]^{-1}\left(\overline{\boldsymbol{x}}-\boldsymbol{\mu}_{0}\right) .
$$

Por fim, Johnson [6] estabelece que se $X_{1}, X_{2}, \ldots, X_{p}$ são independentes e identicamente distribuídas com $N_{p}(\underline{\mu}, \Sigma)$, para uma observação futura $\underline{X}^{\prime}$ da mesma distribuição então

$$
T^{2}=\frac{m}{m+1}(X-\bar{X})^{\prime}[S]^{-1}(X-\bar{X})
$$

tem distribuição

$$
\frac{(m-1) p}{m-p} F_{\alpha, p, m-p}
$$

Neste trabalho, seguindo a indicação de Tracy [10] apud Montgomery [7], o limite superior de controle (LSC) é calculado se baseando numa distribuição beta, em que

$$
L S C=\frac{(m-1)^{2}}{m} \beta_{\alpha, \frac{p}{2}, \frac{m-p-1}{2}}
$$

e o elipsoide $p$ dimensional de $100(1-\alpha) \%$ de previsão de uma futura observação é dado por todos os vetores $\boldsymbol{x}$ satisfazendo

$$
(\boldsymbol{x}-\overline{\boldsymbol{x}})^{\prime}[S]^{-1}(\boldsymbol{x}-\overline{\boldsymbol{x}}) \leq L S C
$$

onde $m$ é a quantidade de amostras (tempo). Em outras palavras, o valor acima é o limite superior de controle (LSC) para o gráfico de controle multivariado $p$ dimensional da estatística $T^{2}$ de Hotelling.

Yu et al. [11] utilizaram esta ideia para o monitoramento de segurança de barragem estabelecendo elipsoide de confiança para análise e previsão aplicado a um estudo de caso na Chencun Hydropower Station, localizada na China.

O relatório [1] afirma que os valores de controle para a leitura da instrumentação da barragem de Itaipu foram estabelecidos com base no método de elementos finitos, modelos reduzidos estruturais e geomecânicos e critérios de projeto. Além disso, os valores obtidos de análises teóricas ou modelos físicos são baseados em certos parâmetros e hipóteses simplificadoras, salientando ainda que deve haver a permanente confrontação entre os valores estabelecidos no projeto com aqueles obtidos nas leituras, não impedindo que eventuais leituras ultrapassem os limites de projeto. Na figura 1 é exibida uma visão geral de Itaipu.

Esta ideia é confirmada por Montgomery [7] e Chaves Neto [3], ou seja, que existem os chamados limites de projeto estabelecidos, mas que pode haver um ajuste destes limites, constituindo os denominados limites de operação estabelecidos com base, por exemplo, em critérios estatísticos, construídos em um período em que a operação da barragem tenha sido considerada normal ou estável.

Para este trabalho, foram selecionados quatro piezômetros para análise. Os piezômetros são localizados no trecho $\mathrm{E}$ da barragem da usina hidrelétrica de Itaipu, a maior geradora de energia elétrica do mundo, localizada no Rio Paraná, entre o Brasil e o Paraguai.

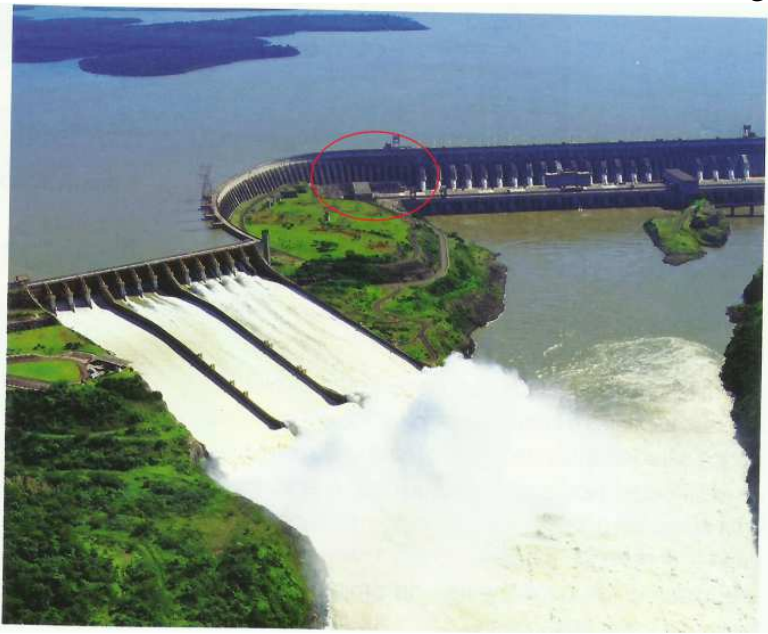

Figura 1- Usina Hidrelétrica de Itaipu e o trecho E em destaque 
Os piezômetros são instrumentos responsáveis pela medida das subpressões nas fundações. A tabela 1 a seguir mostra a localização de cada piezômetro utilizado. Tabela 1 - Localização dos Piezômetros

\begin{tabular}{|l|c|c|c|c|}
\hline \multirow{2}{*}{ Piezômetro } & \multirow{2}{*}{ Feição } & \multicolumn{2}{c|}{ Localização } & \multirow{2}{*}{ Nível } \\
\cline { 3 - 5 } & & Elevação $(\mathrm{m})$ & Afastamento(m) & piezométrico (m) \\
\hline PS-E-26 & Junta D & 124 & -7 & 140 \\
\hline PS-E-27 & Brecha C & 115 & -7 & 140 \\
\hline PS-E-28 & Derrame D & 130 & -53 & 220 \\
\hline PS-E-29 & Junta D & 124 & -53 & 220 \\
\hline
\end{tabular}

Fonte: Relatório [1]

Foram recebidos os dados de leitura manuais destes piezômetros desde a fase de enchimento do reservatório em 1981 até os dias atuais. Alguns dados foram excluídos em função da mudança da periodicidade de leitura que ocorreu com o passar do tempo e da fase de maior variação das leituras, que ocorreu após o enchimento do reservatório. Desta forma, resolveu-se trabalhar com os dados a partir do ano de $2001 \mathrm{em}$ diante. Isto resultou num montante de 316 leituras para cada instrumento. A figura 2 apresenta uma seção do trecho instrumentado (E6) do bloco E e a posição dos quatro instrumentos selecionados.

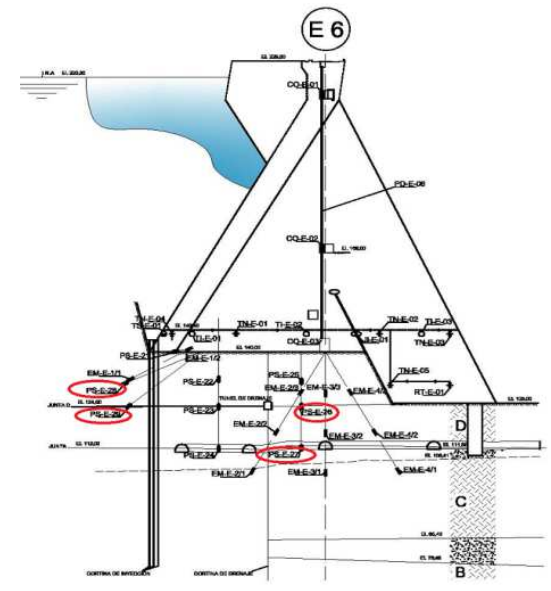

Figura 2 - Instrumentação do bloco E6 em Itaipu

Foram realizados testes de normalidade univariada de Shapiro-Wilk e KolmogorovSmirnov para os dados de cada um dos quatro piezômetros utilizados e o teste de normalidade das amostras bivariadas por qui-quadrado para cada par de piezômetros, $(\boldsymbol{x}-\overline{\boldsymbol{x}})^{\prime}[S]^{-1}(\boldsymbol{x}-$ $\overline{\boldsymbol{x}}) \leq \chi_{2}^{2}$. Os cálculos foram realizados no software Statgraphics.

Para os gráficos bivariados a matriz de covariância foi estimada a partir dos dados agrupados, isto é, pela fórmula

$$
s_{j k}=\frac{1}{n-1} \sum_{i=1}^{n}\left(x_{i j}-\bar{x}_{j}\right)\left(x_{i k}-\bar{x}_{k}\right)
$$

e o limite superior de controle dado pela expressão (2), tal escolha é justificada pois assim o gráfico de controle se torna menos sensível a possíveis mudanças na média, o que compensa o fato de que a variação da temperatura e do nível do reservatório não foi levada em conta neste trabalho.

\section{Resultados}

Os testes de normalidade univariada de Shapiro-Wilk e Kolmogorov-Smirnov apontaram para a rejeição da hipótese de normalidade, mas os testes Qui-quadrado para normalidade bivariada apontaram para a aceitação da hipótese de normalidade, isto se deve ao fato dos testes univariados serem bastante sensíveis e a quantidade de dados disponíveis. Por outro lado, os histogramas não apresentaram forte tendência à anormalidade dos dados e conforme Burr [2] 
apud Montgomery [7], nestes casos os limites de controle baseados na presunção da normalidade são igualmente robustos e aplicáveis aos dados. Quanto aos testes de normalidade bivariada aplicada foram obtidos os seguintes resultados:

Tabela 2: Resultados do teste Qui-quadrado aplicados aos pares de piezômetros

\begin{tabular}{|c|c|c|}
\hline Piezômetros & $\%$ dados $\leq \chi_{2,90 \%}^{2}$ & Correlação \\
\hline 26 e 27 & 87 & 0,4681 \\
\hline 26 e 28 & 92,1 & 0,4168 \\
\hline 26 e 29 & 93,4 & 0,4440 \\
\hline 27 e 28 & 90,8 & 0,6397 \\
\hline 27 e 29 & 91,8 & 0,7098 \\
\hline 28 e 29 & 94,9 & 0,8586 \\
\hline
\end{tabular}

Fonte: Análise dos dados.

Os resultados mostram que os pares de piezômetros se ajustam bem a distribuições normais bivariadas e apresentam de moderada a alta correlação entre os pares $(27,28),(27,29)$ e $(28,29)$ e correlação média entre o piezômetro 26 e os demais. Isto se deve a sua localização de monitoramento.

Após os testes de normalidade foram construídos os gráficos de controle $\bar{x}$ univariados para cada um dos 4 piezômetros. Estes gráficos tem como valor central a média amostral $\bar{x} \mathrm{e}$ como limite superior e inferior de controle, respectivamente, $\bar{x}+3 \sigma$ e $\bar{x}-3 \sigma$ onde $\sigma$ é o desvio padrão amostral. A unidade dos gráficos é msnm (metros sobre nível do mar).

Tabela 3: Quantidade de dados que não caíram dentro da faixa de controle dos gráficos $\bar{x}$

\begin{tabular}{|c|c|}
\hline Piezômetro & Quantidade dados $\notin(\bar{x}-3 \sigma, \bar{x}+3 \sigma)$ \\
\hline PS-E-26 & 83 \\
\hline PS-E-27 & 152 \\
\hline PS-E-28 & 196 \\
\hline PS-E-29 & 168 \\
\hline
\end{tabular}

Fonte: Análise dos dados.

Nota-se que há uma grande quantidade de dados fora dos limites de controle do gráfico de controle, por exemplo, na figura 3 ao observar o gráfico de controle da média amostral de PS-E-28 observa-se uma variação que, segundo a equipe de engenharia da usina é devida à temperatura (causa atribuível), bem como o nível do reservatório, isto se explica pelo fato de não haver nenhum tratamento prévio de fatores ambientais.

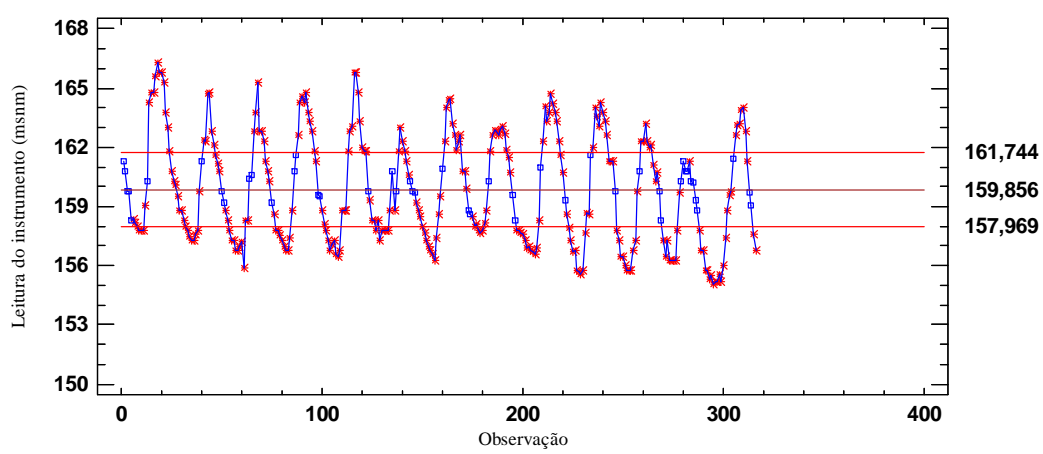

Figura 3 - Gráfico de Controle univariado da média amostral para PS-E-28

Na figura 3 tem-se na linha central a média $\bar{x}$ das leituras e em vermelho os limites inferior de controle $\bar{x}-3 \sigma$ e superior de controle $\bar{x}+3 \sigma$.

Por fim, foram construídos os gráficos de controle bivariados $T^{2}$ de Hotelling para monitorar as médias dos pares de instrumentos. A tabela 4 a seguir resume os resultados obtidos nos gráficos bivariados. Os limites de controle foram estipulados de modo a fornecer uma taxa 
de falsos alarmes de $\alpha=0,27 \%$, para facilitar a comparação com os gráficos univariados. A tabela 4 apresenta também a quantidade de dados (do total de 316) acima do limite superior de controle (LSC) e o resultado do teste de hipótese se os dados podem ser declarados ou não estar dentro do controle estatístico do processo ao nível de $90 \%$ de confiança.

Tabela 4: Resultados do Teste $T^{2}$ de Hotelling para gráficos de controle bivariados

\begin{tabular}{|c|c|c|c|c|c|}
\hline Piezômetros & Dados acima do LSC & Controle & Piezômetros & Dados acima do LSC & Controle \\
\hline PS-E-26 e PS-E-27 & 6 & Não & PS-E-27 e PS-E-28 & 1 & Sim \\
\hline PS-E-26 e PS-E-28 & 6 & Não & PS-E-27 e PS-E-29 & 2 & Sim \\
\hline PS-E-26 e PS-E-29 & 7 & Não & PS-E-28 e PS-E-29 & 2 & Sim \\
\hline
\end{tabular}

Fonte: Análise dos dados.

É exibido na figura 4 o gráfico de controle $T^{2}$ de Hotelling e na figura 5 a elipse de controle do par de piezômetros PS-E-27 e PS-E-28 utilizados neste trabalho.

$\operatorname{LSC}=11,65$

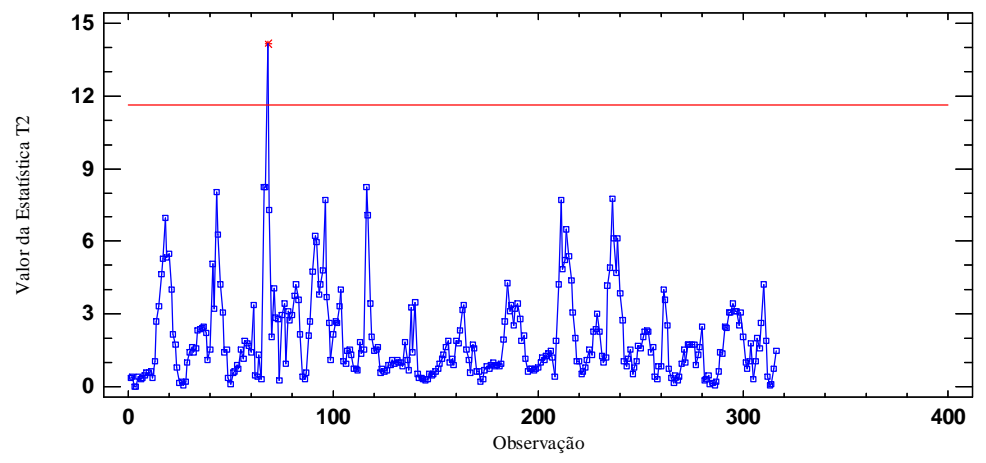

Figura 4 - Gráfico de controle T2 para PS-E-27 e PS-E-28

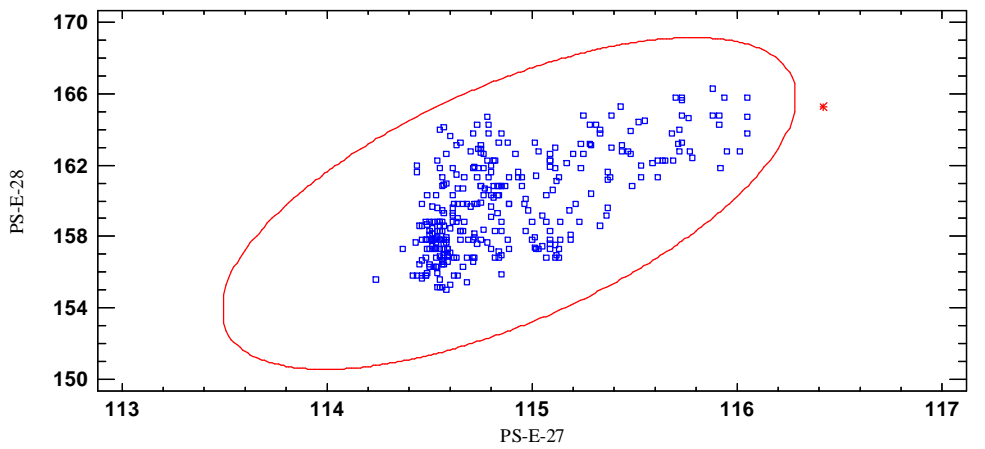

Figura 5 - Elipse de controle para PS-E-27 e PS-E-28

O gráfico $T^{2}$ de Hotelling e a elipse de controle para os piezômetros PS-E-27 e PS-E-28 mostram que somente uma observação (dado) caiu fora dos limites de controle e isto significa que se pode aceitar a hipótese de que estes piezômetros estão em estado de controle estatístico ao nível de $90 \%$ de confiança. 


\section{Conclusões}

As técnicas estatísticas de gráficos de controle, sobretudo os multivariados, para a avaliação do estado de controle de instrumentos de monitoramento de barragens se mostraram eficientes na redução da taxa de alarmes falsos e a indicação de que há forte correlação entre alguns instrumentos. Desta forma, sugere-se a utilização futura de outros gráficos de controle existentes na literatura.

A redução da taxa de falsos alarmes no caso bivariado facilitou a identificação de outliers ou dados discrepantes. Com um número bem menor de pontos fora de controle, pode se observar que estes pontos estão associados às variações térmicas e ao nível de variação do reservatório que necessitam ser incluídas na análise.

Os gráficos de controle bivariados foram capazes de minimizar o efeito da temperatura sobre a análise do estado de controle do monitoramento do instrumento em relação às cartas univariadas, além disso, indicar que se este efeito da temperatura puder ser avaliado (quantificado) e 'retirado' da instrumentação então as cartas bivariadas indicarão apenas um ruído nas leituras e o controle estatístico da instrumentação poderá ser refinado.

Outra colaboração deste trabalho é no sentido de estabelecer limites operacionais para os instrumentos por meio dos gráficos $T^{2}$ de Hotelling e as elipses de controle e avaliar se observações futuras estão sob controle.

\section{Referências Bibliográficas}

[1] Aproveitamento Hidrelétrico de Itaipu - Relatório - Síntese das principais informações de projeto para o controle da instrumentação da barragem principal (trechos E, F, I), 1984.

[2] Burr, I. J. The effect of nonnormality of constants for $\bar{x}$ and $R$ charts. Industrial quality control, vol. 23, 1967.

[3] Chaves Neto, A. Técnicas estatísticas aplicadas à engenharia de qualidade (apostila). Curitiba-PR: UFPR, 2013.

[4] Cheng, L., Zheng D. Two online dam safety monitoring models based on the process of extracting environmental effect. Advances in Engineering Software, 57 (2013), 48-56.

[5] Gu, CS. et al. Singular value diagnosis in dam safety monitoring effect values. Sci China Tech Sci, 2011, 54: 1169-1176.

[6] Johnson, R.A., Wichern, D. W. Applied Multivariate Statistical Analysis (6a ed.). Upper Saddle River-NJ: Pearson Prentice Hall, 2007.

[7] Montgomery, D. C. Introdução ao controle estatístico de qualidade. Rio de Janeiro: LTC, 2013.

[8] Myers, B. Optimization of dam monitoring systems: review of the available technology and case studies. $20^{\text {th }}$. ICOLD International Congress of Large Dams, Beijing, China, 2000.

[9] Ryan, T. P. Statistical Methods for Quality Improvement. New York: J. Wiley, 2011, $3^{\text {rd }}$. ed.

[10] Tracy, N.D.; Young, J.C.; Mason, R.L. Multivariate control charts for individual observations. Journal of quality technology, vol. 24, 1992.

[11] Yu, H, et al. Multivariate analysis in dam monitoring with PCA. Sci China Tech Sci, 2010, 53: 1088-1097. 